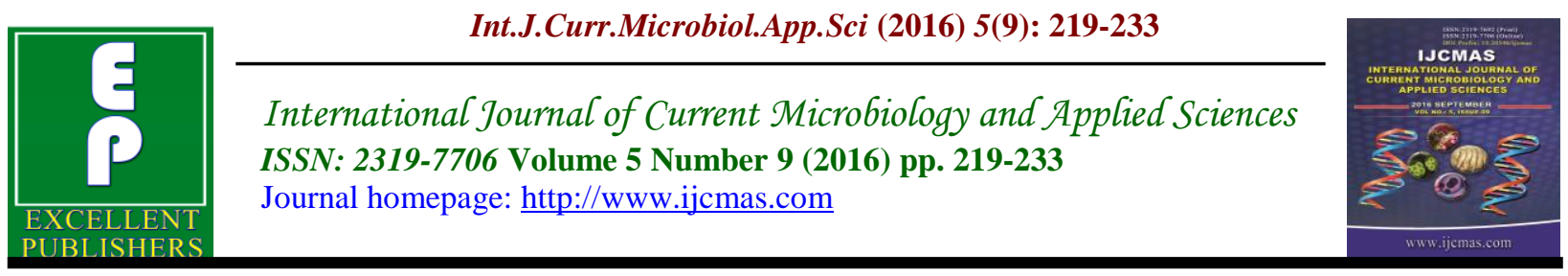

Original Research Article

http://dx.doi.org/10.20546/ijcmas.2016.509.025

\title{
Morphology and Anatomy of Dwarf Shoots of Some Exotic Species of Pinus Linn. in Kumaon Hills, Western Himalayas
}

\author{
Dildar Husain* \\ Department of Botany, University of Lucknow, Lucknow - 226007, India \\ *Corresponding author
}

\section{Keywords \\ Pinus, \\ Dwarf Shoots, \\ Exotic Species \\ Kumaon Hills}

\section{Article Info}

Accepted:

13 August 2016

Available Online:

10 September 2016

\section{A B S T R A C T}

A study was conducted on the Morphology and Anatomy of dwarf shoots of some exotic species of Pinus Linn. In Kumaon Hills, Western Himalayas" and hence morphological and anatomical details of the Pinus growing in above mentioned area. These 5 species of Pinus were collected from different localities of Kumaon Himalayas including Nainital, Ranikhet, Almora, Kaushani and neighbouring areas in Western Himalayas, India. The five species of Pinus considered for the following study included P.canariensis C.Smith (three needles per fascicle), $P$. densiflora Siebold et Zuccarini (two needles per fascicle), P. engelmanii Carriere (three needles per fascicle), $P$. echinata Miller (three needles per fascicle) and $P$. gregii Engelmann (two needles per fascicle). The sections of the dwarf shoots of these species were taken from three different regions and their anatomical details such as shape of epidermal cells, number and position of resin ducts and ratio of cortex to wood were compared. These show interrelationships among different species using morphological, anatomical data. The anatomical details of the Pinus species and their correlation may be incorporated along with other diagnostic features needed for the identification and classification or systematic of these species. This work also attempts to provide a database for the systematics of different species which can be very useful for interspecific comparisons within the same territory and among different territories. The study revealed that anatomical variations in dwarf shoots can be taxonomically very helpful in identification of various species of Pinus.

\section{Introduction}

The genus Pinus Linn. belonging to family Pinaceae is widely distributed and about 105 species have been reported worldwide (Mehra, 1988). Pinus is most dominant genus of the family Pinaceae with its wide range of distribution and adaptability to diverse climatic conditions in Northern as well as Southern Hemisphere on the globe.
In an Asiatic continent, Pinus are distributed mainly in Afghanistan, Pakistan, India, Myanmar, Philippines and spread upto Indonesia after crossing the equator. In India, Kalika forest of Uttarakhand has the largest Pinatum of India where several species of Pinus have been introduced which are flourishing and surviving. 
Commercially, numbers of Pinus species are the main source of timber, paper, herbal products, scents, etc. and various cottage as well as large scale industrial works on based upon Pinus throughout the world. The geographical area of the state is 53,485 sq. kms. The major part of the state of Uttarakhand is hilly. Geographically it is situated in the central Himalayan zone. It extends between $77^{\circ} 34^{\prime}$ and $81^{\circ} 02^{\prime}$ East longitude and $28^{0} 43^{\prime}$ to $31^{0} 27^{\prime}$ North latitude. The state comprises 34 districts. The topography of the Uttarakhand region is characterized by hilly terrain, rugged and rockey mountains, deep valleys, high peaks, sharp streams and rivulets, rapid soil erosion, frequent landslides and widely scattered habitations. The topography of Uttarakhand presents sloppy lands with poor soil depth and fragile ecosystem.

Amongst 105 species of the genus Pinus (Mehra, 1988), only six species are indigenous to Indian flora. These species are Pinus insularis Endlicher, Pinus roxburghii Sargent, Pinus wallichiana A.B. Jackson, Pinus gerardiana Wall ex Lamb., Pinus merkusii Jungh et de Varies and Pinus armendii French. However, the Western Himalayan region of India particularly Kumaon Hills are predominantly covered with various numbers of more other Pinus species which are introduced and these species have established over there.

The study of the dwarf shoot of the genus Pinus dates back since the time of Henry (1837), who described and accurately illustrated and the detail of dwarf shoot organography and certain aspects of its development. Later on, Geylor (1867) work on dwarf shoot was concerned and vascularization of the needles. Subsequently, Schneider (1913) described anatomy of the cataphylls and recognized the spiral arrangement of the needles. The investigation of Borthwick (1899a), Thomson (1914), Prat (1930) were concerned largely with the proliferative capacity of the dwarf shoot. Although the anatomical features of the foliage leaves have been in extensive use in the classification of conifers and other vascular plant groups (Durell, 1916; Harlow, 1931; Fulling, 1934; Marco, 1939; Florin, 1940; Orr, 1944; Buchholz and Gray, 1948; Florin, 1951, 1958, 1963). Sacher (1954) has an intensive study has been made of the structure and seasonal periodicity of the shoot apices of Pinus lambertiana and Pinus ponderosa. Later on, Sacher (1955), Doak (1935) stated that the ontogenetic fate of dwarf shoot apices probable various considerably from species to species. Konar (1963) investigated the morphology and anatomy of Pinus roxburghii. Ghouse and Yunus (1972) was designed and aimed to evolve a simple and workable key for the easy identification of Indian conifers based on their microscopic features of foliage leaves. Debazac (1962), Hanawa (1967), Gabilo and Mogenson (1973) regarded dwarf shoot as microscopic bud and found that the dwarf shoot apex is obliterated during needle development. Owens and Molder (1977) studied on Pinus longaeva and revealed that the long shoot terminal bud is strictly monocyclic. Ewers (1982) stated that microscopic measurement and cell counts revealed that with advancing needle age phloem increases radially and transfusion tissue. Later on, Ewers (1983) studied on the dwarf shoot of Pinus longaeva and stated that the distal dwarf shoot of each flush have interfoliar buds which often proliferate into long shoots. Ewers and Schmid (1985) stated that the dwarf shoot of Pinus longaeva are initiated in the axil of scale leaves in the long shoot terminal bud. Ewers and Aloni (1987) examined that the current year's needles, 1 year old and 2 year old needles of Pinus 
strobus and Pinus brutia and more detailed seasonal analysis of vascular differentiation. Lee and Eom (1988) described and compared the qualitative and quantitative anatomical aspects of dwarf shoots of Pinus koraiensis. Chaturvedi (1993) studied the epidermal and anatomical characters in the needle of two species of Pinus viz. $P$. radiata Don and Pinus yunnanensis Frenchet. Eom and Chung (1994) studied anatomy of tumor wood and normal wood in a branch of Korean red pine (Pinus densiflora Siebold et Zuccarini). Reich et al., (1996) discussed the phenotypic responses in Pinus sylvestris L. and Picea abies L. Lim and Soh (1997) concluded that tracheids in the annual rings of dwarf trees are shorter, narrower and fewer than those of normal trees. Simcha Lev-Yadun (1998) studied relationship between growth ring width and ray size and number. Hafren et al., (2000) studied that developing xylem and mature xylem of Pinus sylvestris is affected by Homogalacturonan. Boratynska and Boratynski (2003) studied abnormal three (or more) needle dwarf shoots of most southern and central European two-needle pine (Pinus) species. They also discussed frequencies of abnormal dwarf shoots in Pinus mugo and Pinus uncinata.

Later on, Donaldson et al., (2004) studied two trees of radiata pine and examined that the presence of compression wood, lignin distribution, microfibril angle, basic density, radial and tangential lumen diameter and cell wall thickness. Esteban et al., (2005) described the anatomical description of the wood as well as an interpretation of the factors related to this resinification. Heuret et al., (2006) discussed the morphological features of wood of Pinus pinaster. Subsequently, Zhang et al., (2008) discussed the formation and significance of radial resin canals in Pinus densiflora. Nair et al., (2009) examined that rays and resin canals were the initiation sites of checking. Boratynski et al., (2011) studied the threeneedle dwarf shoots of Pinus mugo and these were affected by climatic factors. Antony et al., (2012) studied and described different methods used to define the transition from early wood to late wood in Pinus taeda.

The purpose of the present study was conducted on the morphology and anatomy of the dwarf shoots of some exotic species of Pinus. Their anatomical variations was analysed and inter-relationships among the species.

\section{Materials and Methods}

The work deals with the "Morphology and Anatomy of dwarf shoots of some exotic species of Pinus Linn. in Kumaon Hills, Western Himalayas" and hence morphological and anatomical details of the Pinus growing in above mentioned area. These 5 species of Pinus were collected from different localities of Kumaon Himalayas including Nainital, Ranikhet, Almora, Kaushani and neighbouring areas in Western Himalayas, India.

During the present study morphological and anatomical aspects of some exotic species of $P$. have been taken into consideration which constitutes an important aspect of the taxonomical characters of gymnosperms.

The dwarf shoots of some exotic species of Pinus were taken in order to study the anatomical details. They are:

1. Pinus canariensis Smith

2. Pinus densiflora Siebold et Zuccarini

3. Pinus echinata Miller

4. Pinus engelmanii Carriere

5. Pinus greggii Engelmann 
Formalin-propionic acid - Ethyl alcohol was used as a killing and fixation fluid followed by dehydration with the tertiary butyl alcohol method and embedded in Tissuemat. Safrannin staining technique was also employed followed by the use of fast green solution (Johansen, 1940).

All the materials were sectioned upto $8 \mu \mathrm{m}$ in thickness with a razor, then mounted and stained in safrannin and fast green. The microphotographs of transverse sections of dwarf shoots from different regions investigated (taken with help of Nikon Binocular Compund Microscope, FX35DX).

\section{Results and Discussion}

Pinus canariensis Smith commonly known as Canary Island Pine is a large tree ranges from 80-100 feet in height and 10 feet or more in girth (Plate 1, Fig. 1). The branches spreading with drooping blanchlets forming a tree of somewhat weeping habit. Bark thick, reddish, slightly fissured, dividing into irregular scales. Young shoots yellow, winter buds large, ovoid with lance-shaped. Leaves (needles) in threes per fascicle, highly glaucous on seedling (Dallimore and Jackson, 1966). The spur length may vary, it is upto 4.2 inch and dwarf shoot about $1 \mathrm{~cm}$ in length (Plate 1, Fig. 2).

Pinus densiflora Siebold et Zuccarini commonly known as Japanese Red Pine is a large tree ranges from 70-120 feet in height and 6-12 feet in girth (Plate 1, Fig. 6). Bark reddish like that of Scots pine, particularly about the higher parts of tree, peeling off in thin scales. Young shoots green with a glaucous bloom. Winter buds, slender, cylindrical with sharp pointed. Leaves (needles) in pairs per fascicle persisting three years (Dallimore and Jackson, 1966). The spur length may vary, it is upto 3.5 inch and dwarf shoot about $1 \mathrm{~cm}$ in length (Plate 1, Fig. 7).
Pinus echinata Miller commonly known as short leaf pine is a large tree ranges from 80100 or occasionally 120 feet in height with a trunk 4-6 feet in girth (Plate 1, Fig. 11). The bark thick, reddish divided into irregular shaped. Young shoots pale green flushed with violet and usually coated with a glaucous bloom changing to brown. Winter buds ovate, sharp pointed. Leaves (needles) usually in pairs but occasionally in threes or fours also have been reported (Dallimore and Jackson, 1966). The spur length may vary, it is upto 3.7 inch and dwarf shoots about $9 \mathrm{~mm}$ in length (Plate 1, Fig. 12).

Pinus engelmanii Carriere commonly known as Apache pine is a medium sized tree ranges from 20-30 meters in height and a trunk diameter of 35-80 cm (Plate 2, Fig. 1). Bark dark brown, at maturity deeply furrowed. Branches straight to ascending. Winter buds ovoid to conic, resinous. Leaves (needles) usually in threes, often fives per fascicle, persisting two years (Dallimore and Jackson, 1966). The spur length may vary, it is upto 7.8 inch and dwarf shoot about $1 \mathrm{~cm}$ in length (Plate 2, Fig. 2).

Pinus greggii Engelmann commonly known as Gregg's pine is a medium size tree ranges from 40-50 feet in height with smooth grey bark except at the base of old trees where it becomes rough and fissured (Plate 2, Fig. 6). Young shoots glaucous at first, greyish-buff the second year. Winter buds slender, cylindrical with sharp-pointed. Leaves (needles) are usually in threes but rarely two in each fascicle, persisting two-three years (Dallimore and Jackson, 1966). The spur length may vary, it is upto 8.2 inch and dwarf shoot about $1 \mathrm{~cm}$ in length (Plate 2, Fig. 7).

The sections of the dwarf shoots of above mentioned species were taken from three 
different regions and their anatomical details were compared. Many variations were recorded in the anatomical structures of dwarf shoots such as shape of epidermal cells, number and position of resin ducts, ratio of cortex to wood, etc.

The basal region of dwarf shoot was bounded by membranous sheath formed by scale leaves instead of a well defined epidermis. Foster's (1938) opinion that tunica and corpus represent interdependent growth zones in apical meristem and as such, one cannot exist independently of the other. P. canariensis (Plate 1, Fig. 3) has very thick walled cuticle covered with scales leaves which shows similarities with $P$. densiflora (Plate 1, Fig. 8), P. echinata (Plate 1, Fig. 13) and P. greggii (Plate 2, Fig. 8) while $P$. engelmanii shows thick walled cuticle but some cells constitute the epidermis (Plate 2, Fig. 3).

The cortical region may be differentiated into two zones viz. outer and inner zone. The outer zone consists of parenchymatous cells, six to seven layered in $P$. canariensis (Plate 1, Fig. 3), four to five layered in $P$. densiflora (Plate 1, Fig. 8), five to six layered in P. echinata (Plate 1, Fig. 13) and $P$. greggii (Plate 2, Fig. 8) while in $P$. engelmanii (Plate 2, Fig. 3), these are six to seven layered.

The shape and size of outer zone of cortical cells may also vary. The outer cortical cells are oval, elongated, quadrangular, penta to hexagonal in $P$. canariensis (Plate 1, Fig. 3) and elongated, quadrangular, penta to hexagonal in $P$. densiflora (Plate 1, Fig. 8) which shows similarity with $P$. echinata (Plate 1, Fig. 13) where these are oval, quadrangular, penta to hexagonal. $P$. engelmanii (Plate 2, Fig. 3) has oval, elongated, quadrangular to pentagonal while in P. greggii (Plate 2, Fig. 8), these are elongated, quadrangular to pentagonal. The size of outer cortical cells may also varied, 22.2-44.4 $\mu \mathrm{m}$ in long and 11.1-33.3 $\mu \mathrm{m}$ in broad in $P$. canariensis (Plate 1, Fig. 3, Table 1), 11.1-44.4 $\mu \mathrm{m}$ in long and 11.1-33.3 $\mu \mathrm{m}$ broad in $P$. densiflora (Plate 1, Fig. 8, Table 1) and $P$. engelmanii (Plate 2, Fig. 3, Table 1), 11.1-33.3 $\mu \mathrm{m}$ in long and 11.1-27.7 $\mu \mathrm{m}$ in broad in $P$. echinata (Plate 1, Fig. 13, Table 1) and 22.2-55.5 $\mu \mathrm{m}$ in long and $11.1-33.3 \mu \mathrm{m}$ in broad in P. greggii (Plate 2, Fig.8,Table 1).

Resin ducts are absent in this zone in case of P. canariensis (Plate 1, Fig. 3), P. densiflora (Plate 1, Fig. 8) and P. greggii (Plate 2, Fig. 8) while these are present in $P$. echinata (Plate 1, Fig. 13) and P. engelmanii (Plate 2, Fig. 3). In P. echinata (Plate 1, Fig. 13), the resin ducts distributed irregularly here and there, lined by thin walled, single layered of epithelial cells, placed intermarginally while in P. engelmanii (Plate 2, Fig. 3), these are also thin walled but two layered of epithelial cells, placed intermarginally. The cells of inner zone of cortex are relatively smaller, thin walled than the outer zone of cortical cells.

The inner zone also consists of parenchymatous cells, thin walled, small, loosely or compactly arranged. These are five to six layered in $P$. canariensis (Plate 1, Fig. 3), three to four layered in $P$. densiflora (Plate 1, Fig. 8), P. echinata (Plate 1, Fig. 13), $P$. engelmanii (Plate 2, Fig. 3) and $P$. greggii (Plate 2, Fig. 8). The shape and size of inner cortical cells may also vary. These are elongated, quadrangular to pentagonal in $P$. canariensis (Plate 1, Fig. 3), P. densiflora (Plate 1, Fig. 8) and P. echinata (Plate 1, Fig. 13). These shows much affinity with $P$. engelmanii (Plate 2, Fig. 3) and P. greggii (Plate 2, Fig. 8) which contain oval, elongated, quadrangular to pentagonal cells. 
Table.1 Anatomical details of dwarf shoots at three different regions viz. basal, middle and upper

\begin{tabular}{|c|c|c|c|c|c|c|c|c|c|c|c|c|c|c|c|c|}
\hline \multirow{4}{*}{$\begin{array}{l}\text { SL. } \\
\text { NO. }\end{array}$} & \multirow{4}{*}{$\begin{array}{l}\text { NAME OF } \\
\text { SPECIES }\end{array}$} & \multirow{4}{*}{ CUTICLE } & \multicolumn{14}{|c|}{ BASAL REGION } \\
\hline & & & \multicolumn{2}{|c|}{ EPIDERMIS } & \multicolumn{2}{|c|}{ HYPODERMIS } & \multicolumn{6}{|c|}{ CORTICAL ZONE } & \multicolumn{3}{|c|}{ WOOD REGION } & \multirow{3}{*}{$\begin{array}{c}\text { PITH } \\
\text { REGION }\end{array}$} \\
\hline & & & \multirow{2}{*}{ SHAPE } & \multirow{2}{*}{ SIZE } & \multirow{2}{*}{ SHAPE } & \multirow{2}{*}{ SIZE } & \multicolumn{3}{|c|}{ OUTER ZONE } & \multicolumn{3}{|c|}{ INNER ZONE } & \multirow{2}{*}{ AMOUNT } & \multirow{2}{*}{ SIZE } & \multirow{2}{*}{ R.D. } & \\
\hline & & & & & & & SHAPE & SIZE & R.D. & SHAPE & SIZE & R.D. & & & & \\
\hline 1. & $\begin{array}{l}\text { P. canariensis } \\
\text { Smith } \\
\text { (3 needles per } \\
\text { fascicle) }\end{array}$ & $\begin{array}{l}\text { Scale } \\
\text { leaves }\end{array}$ & ND & ND & ND & ND & $\begin{array}{c}\text { Oval, } \\
\text { elongated } \\
\text { quadrangular, } \\
\text { penta to } \\
\text { hexagonal }\end{array}$ & $\begin{array}{c}\text { 22.2-44.4 } \mu \mathrm{m} \\
\text { long \& } 11.1- \\
33.3 \mu \mathrm{m} \\
\text { broad }\end{array}$ & - & $\begin{array}{l}\text { Elongated, } \\
\text { quadrangular } \\
\text { to pentagonal }\end{array}$ & $\begin{array}{c}11.1-22.2 \\
\mu \mathrm{m} \text { long \& } \\
11.1-16.6 \\
\mu \mathrm{m} \text { broad }\end{array}$ & + & $\begin{array}{l}\text { Less in } \\
\text { amount as } \\
\text { compared } \\
\text { to the } \\
\text { amount of } \\
\text { cortex }\end{array}$ & \begin{tabular}{|c|}
$321.9-333$ \\
$\mu \mathrm{m}$ long \& \\
$277.5-288.6$ \\
$\mu \mathrm{m}$ broad
\end{tabular} & + & $\begin{array}{c}\text { Centrally } \\
\text { located \& } \\
\text { less in } \\
\text { amount }\end{array}$ \\
\hline 2. & $\begin{array}{l}\text { P. densiflora } \\
\text { Siebold et. } \\
\text { Zuccarini } \\
\text { (2 needles per } \\
\text { fascicle) }\end{array}$ & $\begin{array}{l}\text { Scale } \\
\text { leaves }\end{array}$ & ND & ND & ND & ND & $\begin{array}{c}\text { Elongated } \\
\text { quadrangular, } \\
\text { penta to } \\
\text { hexagonal }\end{array}$ & \begin{tabular}{|c|}
$11.1-44.4 \mu \mathrm{m}$ \\
long $\& 11.1-$ \\
$33.3 \mu \mathrm{m}$ \\
broad
\end{tabular} & - & $\begin{array}{l}\text { Elongated, } \\
\text { quadrangular } \\
\text { to pentagonal }\end{array}$ & $\begin{array}{c}11.1-16.6 \\
\mu \mathrm{m} \text { long \& } \\
11.1-22.2 \\
\mu \mathrm{m} \text { broad }\end{array}$ & + & $\begin{array}{c}\text { Less in } \\
\text { amount as } \\
\text { compared } \\
\text { to the } \\
\text { amount of } \\
\text { cortex }\end{array}$ & \begin{tabular}{|c|}
$266.4-277.5$ \\
$\mu \mathrm{m}$ long \& \\
$244.2-255.3$ \\
$\mu \mathrm{m}$ broad
\end{tabular} & - & $\begin{array}{c}\text { Centrally } \\
\text { located \& } \\
\text { less in } \\
\text { amount }\end{array}$ \\
\hline 3. & $\begin{array}{c}\text { P. echinata } \\
\text { Miller } \\
(2 \text { needles per } \\
\text { fascicle) }\end{array}$ & $\begin{array}{l}\text { Scale } \\
\text { leaves }\end{array}$ & ND & ND & ND & ND & $\begin{array}{c}\text { Oval, } \\
\text { elongated } \\
\text { quadrangular, } \\
\text { penta to } \\
\text { hexagonal }\end{array}$ & \begin{tabular}{|c|}
$11.1-33.3 \mu \mathrm{m}$ \\
long \& $11.1-$ \\
$27.7 \mu \mathrm{m}$ \\
broad
\end{tabular} & + & $\begin{array}{c}\text { Quadrangular, } \\
\text { elongated to } \\
\text { pentagonal }\end{array}$ & $\begin{array}{c}5.55-11.1 \\
\mu \mathrm{m} \text { long \& } \\
5.55-16.6 \\
\mu \mathrm{m} \text { broad }\end{array}$ & - & $\begin{array}{c}\text { Less in } \\
\text { amount as } \\
\text { compared } \\
\text { to the } \\
\text { amount of } \\
\text { cortex }\end{array}$ & $\begin{array}{c}222-233 \\
\mu \mathrm{m} \text { long \& } \\
177-188 \\
\mu \mathrm{m} \text { broad }\end{array}$ & + & $\begin{array}{c}\text { Centrally } \\
\text { located \& } \\
\text { less in } \\
\text { amount }\end{array}$ \\
\hline 4. & $\begin{array}{l}\text { P. engelmanii } \\
\text { Carriere } \\
\text { (3 needles per } \\
\text { fascicle) }\end{array}$ & $\begin{array}{l}\text { Scale } \\
\text { leaves }\end{array}$ & ND & ND & ND & ND & $\begin{array}{c}\text { Oval, } \\
\text { elongated } \\
\text { quadrangular } \\
\text { to pentagonal }\end{array}$ & $\begin{array}{c}11.1-44.4 \mu \mathrm{m} \\
\text { long } \& 11.1- \\
33.3 \mu \mathrm{m} \\
\text { broad }\end{array}$ & - & $\begin{array}{c}\text { Oval } \\
\text { elongated, } \\
\text { quadrangular } \\
\text { to pentagonal }\end{array}$ & $\begin{array}{c}11.1-16.6 \\
\mu \mathrm{m} \text { long \& } \\
11.1-27.7 \\
\mu \mathrm{m} \text { broad }\end{array}$ & - & $\begin{array}{c}\text { Less than } \\
\text { the amount } \\
\text { of cortex }\end{array}$ & $\begin{array}{l}321.9-333 \\
\mu \mathrm{m} \text { long \& } \\
210.9-222 \\
\mu \mathrm{m} \text { broad }\end{array}$ & - & $\begin{array}{l}\text { Less in } \\
\text { amount } \\
\text { and } \\
\text { occupying } \\
\text { minimum } \\
\text { area } \\
\end{array}$ \\
\hline 5. & $\begin{array}{c}\text { P. greggii } \\
\text { Engelmann } \\
(3 \text { needles per } \\
\text { fascicle) }\end{array}$ & $\begin{array}{l}\text { Scale } \\
\text { leaves }\end{array}$ & ND & ND & ND & ND & $\begin{array}{c}\text { Guadrangular, } \\
\text { elongated to } \\
\text { pentagonal }\end{array}$ & \begin{tabular}{|c|}
$22.2-55.5 \mu \mathrm{m}$ \\
long $\& 11.1-$ \\
$33.3 \mu \mathrm{m}$ \\
broad
\end{tabular} & - & $\begin{array}{c}\text { Oval, } \\
\text { elongated, } \\
\text { quadrangular } \\
\text { to pentagonal }\end{array}$ & $\begin{array}{c}11.1-16.6 \\
\mu \mathrm{m} \text { long } \& \\
11.1-22.2 \\
\mu \mathrm{m} \text { broad }\end{array}$ & + & \begin{tabular}{|c|} 
Less than \\
the amount \\
of cortex
\end{tabular} & \begin{tabular}{|c|}
$155.4-166.5$ \\
$\mu \mathrm{m}$ long \& \\
$166.5-177.6$ \\
$\mu \mathrm{m}$ broad
\end{tabular} & - & $\begin{array}{l}\text { Least in } \\
\text { amount } \\
\text { and } \\
\text { occupying } \\
\text { minimum } \\
\text { area }\end{array}$ \\
\hline
\end{tabular}


Table.2

\begin{tabular}{|c|c|c|c|c|c|c|c|c|c|c|c|c|c|c|c|c|c|c|c|}
\hline \multirow{4}{*}{$\begin{array}{l}\text { SL. } \\
\text { NO. }\end{array}$} & \multirow{4}{*}{$\begin{array}{l}\text { NAME OF } \\
\text { SPECIES }\end{array}$} & \multicolumn{18}{|c|}{ MIDDLE REGION } \\
\hline & & \multirow{3}{*}{ CUTICLE } & \multicolumn{2}{|c|}{ EPIDERMIS } & \multicolumn{2}{|c|}{ HYPODERMIS } & \multicolumn{6}{|c|}{ CORTICAL ZONE } & \multicolumn{6}{|c|}{ WOOD REGION } & \multirow{3}{*}{$\begin{array}{c}\text { PITH } \\
\text { REGION }\end{array}$} \\
\hline & & & \multirow{2}{*}{ SHAPE } & \multirow{2}{*}{ SIZE } & \multirow{2}{*}{ SHAPE } & \multirow{2}{*}{ SIZE } & \multicolumn{3}{|c|}{ OUTER ZONE } & \multicolumn{3}{|c|}{ INNER ZONE } & \multicolumn{2}{|c|}{ ENDO. } & \multicolumn{2}{|c|}{ PERICYCLE } & \multicolumn{2}{|c|}{ V.B. } & \\
\hline & & & & & & & SHAPE & SIZE & R.D. & SHAPE & SIZE & R.D. & SHAPE & SIZE & SHAPE & SIZE & SIZE & R.D. & \\
\hline 1. & $\begin{array}{l}\text { P. canariensis } \\
\text { Smith } \\
\text { (3 needles per } \\
\text { fascicle) }\end{array}$ & $\begin{array}{c}\text { Thick } \\
\text { walled }\end{array}$ & $\begin{array}{c}\text { Isodia- } \\
\text { metric, } \\
\text { elongated to } \\
\text { rectangular }\end{array}$ & $\begin{array}{c}11.1 \text { to } \\
16.6 \mu \mathrm{m} \\
\text { long \& } \\
11.1 \text { to } \\
16.6 \mu \mathrm{m} \\
\text { broad }\end{array}$ & ND & ND & $\begin{array}{l}\text { Isodiametric } \\
\text { quadrangular, } \\
\text { elongated to } \\
\text { pentagonal }\end{array}$ & $\begin{array}{c}16.6 \text { to } \\
44.4 \mu \mathrm{m} \\
\text { long \& } \\
11.1 \text { to } \\
16.6 \mu \mathrm{m} \\
\text { broad }\end{array}$ & ND & $\begin{array}{l}\text { Quadrangular, } \\
\text { isodiametric \& } \\
\text { elongated }\end{array}$ & $\begin{array}{c}11.1 \text { to } \\
16.6 \mu \mathrm{m} \\
\text { long \& } \\
11.1 \text { to } \\
16.6 \mu \mathrm{m} \\
\text { broad }\end{array}$ & ND & ND & ND & ND & ND & $\begin{array}{c}111 \text { to } \\
122.1 \\
\mu \mathrm{m} \text { long } \\
\& 177.6 \\
\text { to } 199.8 \\
\mu \mathrm{m} \\
\text { broad }\end{array}$ & + & $\begin{array}{c}\text { Less in } \\
\text { amount } \\
\text { different- } \\
\text { tiated into } \\
\text { three } \\
\text { distinct } \\
\text { zones }\end{array}$ \\
\hline 2. & $\begin{array}{l}\text { P. densiflora } \\
\text { Siebold et. } \\
\text { Zuccarini } \\
\text { (2 needles per } \\
\text { fascicle) }\end{array}$ & $\begin{array}{c}\text { Thin } \\
\text { walled }\end{array}$ & $\begin{array}{c}\text { Oval, } \\
\text { elongated } \\
\text { quadrangular }\end{array}$ & $\begin{array}{c}5.55 \text { to } \\
11.1 \mu \mathrm{m} \\
\text { long \& } \\
5.55 \text { to } \\
16.6 \mu \mathrm{m} \\
\text { broad }\end{array}$ & \begin{tabular}{|c|} 
Oval \\
elongate \\
$\mathrm{d}$ to \\
pentagon \\
al
\end{tabular} & $\begin{array}{c}5.55 \text { to } \\
11.1 \mu \mathrm{m} \\
\text { long \& } \\
5.55 \text { to } \\
16.6 \mu \mathrm{m} \\
\text { broad }\end{array}$ & ND & ND & ND & $\begin{array}{c}\text { Oval, } \\
\text { elongated, } \\
\text { quadrangular, } \\
\text { penta to } \\
\text { hexagonal }\end{array}$ & $\begin{array}{c}11.1 \text { to } \\
33.3 \mu \mathrm{m} \\
\text { long \& } \\
11.1 \text { to } \\
27.7 \mu \mathrm{m} \\
\text { broad }\end{array}$ & + & ND & ND & ND & ND & $\begin{array}{c}99.9 \text { to } \\
111 \mu \mathrm{m} \\
\text { long \& } \\
177.6 \text { to } \\
199.8 \\
\mu \mathrm{m} \\
\text { broad }\end{array}$ & + & \begin{tabular}{|} 
Less in \\
amount \\
different- \\
tiated into \\
three \\
distinct \\
zones
\end{tabular} \\
\hline 3. & $\begin{array}{c}\text { P. echinata } \\
\text { Miller } \\
\text { (2 needles per } \\
\text { fascicle) }\end{array}$ & $\begin{array}{c}\text { Thick } \\
\text { walled }\end{array}$ & \begin{tabular}{|c|} 
Oval \\
isodiametric, \\
quadrangular \\
to elongated
\end{tabular} & $\begin{array}{c}5.55 \text { to } \\
11.1 \mu \mathrm{m} \\
\text { long \& } \\
5.55 \text { to } \\
11.1 \mu \mathrm{m} \\
\text { broad }\end{array}$ & $\begin{array}{l}\text { Oval } \\
\text { elongate } \\
\text { d to } \\
\text { quadra- } \\
\text { ngular }\end{array}$ & $\begin{array}{c}11.1 \text { to } \\
16.6 \mu \mathrm{m} \\
\text { long \& } \\
5.55 \text { to } \\
16.6 \mu \mathrm{m} \\
\text { broad }\end{array}$ & ND & ND & ND & $\begin{array}{c}\text { Isodiametric, } \\
\text { oval, elongated, } \\
\text { quadrangular, to } \\
\text { pentagonal }\end{array}$ & $\begin{array}{c}11.1 \text { to } \\
27.7 \mu \mathrm{m} \\
\text { long \& } \\
11.1 \text { to } \\
27.7 \mu \mathrm{m} \\
\text { broad }\end{array}$ & + & ND & ND & ND & ND & $\begin{array}{c}88.8 \text { to } \\
99.9 \mu \mathrm{m} \\
\text { long \& } \\
188.7 \text { to } \\
199.8 \\
\mu \mathrm{m} \\
\text { broad }\end{array}$ & - & $\begin{array}{l}\text { Less in } \\
\text { amount } \\
\text { and } \\
\text { centrally } \\
\text { located }\end{array}$ \\
\hline 4. & $\begin{array}{l}\text { P. engelmanii } \\
\text { Carriere } \\
\text { (3 needles per } \\
\text { fascicle) }\end{array}$ & $\begin{array}{l}\text { Scale } \\
\text { leaves }\end{array}$ & ND & ND & ND & ND & $\begin{array}{c}\text { Oval, } \\
\text { elongated, } \\
\text { isodiametric, } \\
\text { quadrantular } \\
\text { to hexagonal }\end{array}$ & $\begin{array}{c}11.1 \text { to } \\
44.4 \mu \mathrm{m} \\
\text { long \& } \\
11.1 \text { to } \\
33.3 \mu \mathrm{m} \\
\text { broad }\end{array}$ & + & ND & ND & ND & ND & ND & ND & ND & $\begin{array}{c}111- \\
133.2 \\
\mu \mathrm{m} \text { long } \\
\& 188.7- \\
222 \mu \mathrm{m} \\
\text { broad }\end{array}$ & ND & $\begin{array}{l}\text { Centrally } \\
\text { located } \\
\text { and } \\
\text { occupying } \\
\text { minimum } \\
\text { area }\end{array}$ \\
\hline 5. & $\begin{array}{c}\text { P. greggii } \\
\text { Engelmann } \\
(3 \text { needles per } \\
\text { fascicle })\end{array}$ & $\begin{array}{c}\text { Thin } \\
\text { walled }\end{array}$ & \begin{tabular}{|c|} 
Oval \\
isodiametric, \\
elongated to \\
rectangular
\end{tabular} & $\begin{array}{c}11.1-16.6 \\
\mu \mathrm{m} \text { long \& } \\
11.1-16.6 \\
\mu \mathrm{m} \text { broad }\end{array}$ & $\begin{array}{c}\text { Oval } \\
\text { elongate } \\
\mathrm{d} \text { to } \\
\text { pentagon } \\
\text { al }\end{array}$ & $\begin{array}{c}11.1- \\
16.6 \mu \mathrm{m} \\
\text { long \& } \\
5.55- \\
11.1 \mu \mathrm{m} \\
\text { broad }\end{array}$ & $\begin{array}{c}\text { Oval, } \\
\text { elongated, } \\
\text { quadrantular } \\
\text { to hexagonal }\end{array}$ & $\begin{array}{c}11.1-27.7 \\
\mu \mathrm{m} \text { long \& } \\
11.1-22.2 \\
\mu \mathrm{m} \text { broad }\end{array}$ & + & ND & ND & ND & ND & ND & ND & ND & $\begin{array}{c}66.6- \\
77.7 \mu \mathrm{m} \\
\text { long \& } \\
111- \\
133.2 \\
\mu \mathrm{m} \\
\text { broad }\end{array}$ & + & $\begin{array}{l}\text { Centrally } \\
\text { located } \\
\text { and } \\
\text { occupying } \\
\text { minimum } \\
\text { area }\end{array}$ \\
\hline
\end{tabular}

ENDO $=$ ENDODERMIS; PERI. $=$ PERICYCLE; R.D. $=$ RESIN DUCT; V.B. $=$ VASCULAR BUNDLE; N.D. $=$ NOT DISTINCT; L.D. = LESS DISTINCT; $+=$ PRESENT; $-=$ ABSENT 
Table.3

\begin{tabular}{|c|c|c|c|c|c|c|c|c|c|c|c|c|c|c|c|c|}
\hline \multirow{4}{*}{\begin{tabular}{l|} 
SL. \\
NO.
\end{tabular}} & \multirow{4}{*}{$\begin{array}{l}\text { NAME OF } \\
\text { SPECIES }\end{array}$} & \multicolumn{15}{|c|}{$\begin{array}{l}\text { UPPER REGION } \\
\end{array}$} \\
\hline & & \multirow[t]{3}{*}{ CUTICLE } & \multicolumn{2}{|c|}{ EPIDERMIS } & \multicolumn{2}{|c|}{ HYPODERMIS } & \multirow{2}{*}{\multicolumn{3}{|c|}{$\begin{array}{l}\text { MESOPHYLLS (ARM PALISADE } \\
\& \text { SPOGY PARENCHYMA) \& R.D. }\end{array}$}} & \multicolumn{6}{|c|}{ VASCULAR ZONE } & \\
\hline & & & \multirow{2}{*}{ SHAPE } & \multirow{2}{*}{ SIZE } & \multirow{2}{*}{ SHAPE } & \multirow{2}{*}{ SIZE } & & & & \multicolumn{2}{|c|}{ ENDO. } & \multicolumn{2}{|c|}{ PERICYCLE } & \multicolumn{2}{|l|}{ V.B. } & \\
\hline & & & & & & & \begin{tabular}{|l|} 
SHAPE \\
\end{tabular} & SIZE & R.D. & SHAPE & SIZE & \begin{tabular}{l|l} 
SHAPE & \\
\end{tabular} & Size & Size & R.D. & \\
\hline 1. & $\begin{array}{l}\text { P. canariensis } \\
\text { Smith } \\
\text { (3 needles per } \\
\text { fascicle) }\end{array}$ & $\begin{array}{l}\text { Thin } \\
\text { walled }\end{array}$ & $\begin{array}{l}\text { Oval, } \\
\text { elongated to } \\
\text { rectangular }\end{array}$ & $\mid \begin{array}{c}\text { 5.55-11.1 } \mu \mathrm{m} \\
\text { long \& } 5.55- \\
11.1 \mu \mathrm{m} \\
\text { broad }\end{array}$ & $\begin{array}{c}\text { Oval, } \\
\text { quadrangular, } \\
\text { to elongated }\end{array}$ & $\begin{array}{c}11.1-16.6 \\
\mu \mathrm{m} \text { long } \& \\
11.1-22.2 \\
\mu \mathrm{m} \text { broad }\end{array}$ & $\begin{array}{c}\text { Quadrangular, } \\
\text { oval, } \\
\text { elongated to } \\
\text { pentagonal }\end{array}$ & \begin{tabular}{|c|}
$11.1-$ \\
$22.2 \mu \mathrm{m}$ \\
long \& \\
$16.6-$ \\
$27.7 \mu \mathrm{m}$ \\
broad
\end{tabular} & $\begin{array}{c}+ \\
\& \\
2 \text { in no. in } \\
\text { each zone }\end{array}$ & ND & ND & ND & ND & $\begin{array}{c}77.7-88.8 \\
\mu \mathrm{m} \text { long \& } \\
133.2-144.3 \\
\mu \mathrm{m} \text { broad }\end{array}$ & - & - \\
\hline 2. & $\begin{array}{l}\text { P. densiflora } \\
\text { Siebold et. } \\
\text { Zuccarini } \\
\text { (2 needles per } \\
\text { fascicle) }\end{array}$ & $\begin{array}{c}\text { Thin } \\
\text { walled }\end{array}$ & $\begin{array}{c}\text { Oval, } \\
\text { elongated to } \\
\text { quadrangular }\end{array}$ & $\begin{array}{c}\text { 5.55-11.1 } \mu \mathrm{m} \\
\text { long \& } 5.55- \\
16.6 \mu \mathrm{m} \\
\text { broad }\end{array}$ & $\begin{array}{c}\text { Oval, } \\
\text { elongated, } \\
\text { quadrangular }\end{array}$ & $\begin{array}{c}5.55-11.1 \\
\mu \mathrm{m} \text { long } \& \\
5.55-16.6 \\
\mu \mathrm{m} \text { broad }\end{array}$ & $\begin{array}{c}\text { Less } \\
\text { differentiate, } \\
\text { elongated } \\
\text { (polygonal) }\end{array}$ & \begin{tabular}{|c|}
$11.1-$ \\
$38.8 \mu \mathrm{m}$ \\
long \& \\
$11.1-$ \\
$33.3 \mu \mathrm{m}$ \\
broad
\end{tabular} & $\begin{array}{c}+ \\
\& \\
2 \text { in no. in } \\
\text { each zone }\end{array}$ & ND & ND & ND & ND & $\begin{array}{c}99.9-111 \mu \mathrm{m} \\
\text { long \& } \\
155.4-166.5 \\
\mu \mathrm{m} \text { broad }\end{array}$ & - & - \\
\hline 3. & $\begin{array}{c}\text { P. echinata } \\
\text { Miller } \\
\text { (2 needles per } \\
\text { fascicle) }\end{array}$ & $\begin{array}{l}\text { Thin } \\
\text { walled }\end{array}$ & $\begin{array}{l}\text { Oval to } \\
\text { rectangular }\end{array}$ & $\mid \begin{array}{c}5.55-11.1 \mu \mathrm{m} \\
\text { long \& } 5.55- \\
11.1 \mu \mathrm{m} \\
\text { broad }\end{array}$ & $\begin{array}{l}\text { Oval, } \\
\text { elongated to } \\
\text { rectantular }\end{array}$ & $\begin{array}{c}5.55-11.1 \\
\mu \mathrm{m} \text { long } \& \\
5.55-16.6 \\
\mu \mathrm{m} \text { broad }\end{array}$ & $\begin{array}{c}\text { Oval, } \\
\text { elongated } \\
\text { quadrangular, } \\
\text { circular }\end{array}$ & \begin{tabular}{|c|}
$11.1-$ \\
$27.7 \mu \mathrm{m}$ \\
long \& \\
$11.1-$ \\
$27.7 \mu \mathrm{m}$ \\
broad
\end{tabular} & $\begin{array}{c}+ \\
\& \\
2 \text { in no. in } \\
\text { each zone }\end{array}$ & LD & LD & LD & LD & $\begin{array}{c}88.8-99.9 \\
\mu \mathrm{m} \text { long \& } \\
188.7-199.8 \\
\mu \mathrm{m} \text { broad }\end{array}$ & - & - \\
\hline 4. & $\begin{array}{l}\text { P. engelmanii } \\
\text { Carriere } \\
\text { (3 needles per } \\
\text { fascicle) }\end{array}$ & $\begin{array}{l}\text { Thick } \\
\text { walled }\end{array}$ & $\begin{array}{c}\text { Oval, } \\
\text { isodiametric, } \\
\text { elongated to } \\
\text { rectangular }\end{array}$ & \begin{tabular}{|c|}
$5.55-11.1 \mu \mathrm{m}$ \\
long \& \\
$11.15-16.6$ \\
$\mu \mathrm{m}$ broad
\end{tabular} & $\begin{array}{c}\text { Oval, } \\
\text { elongated to } \\
\text { quadrangular } \\
\text { to pentagonal }\end{array}$ & $\begin{array}{c}5.55-16.6 \\
\mu \mathrm{m} \text { long } \& \\
11.1-16.6 \\
\mu \mathrm{m} \text { broad }\end{array}$ & $\begin{array}{c}\text { Elongated } \\
\text { quadrangular, } \\
\text { penta to } \\
\text { octagonal }\end{array}$ & \begin{tabular}{|c|}
$11.1-$ \\
$22.2 \mu \mathrm{m}$ \\
long \& \\
$16.6-$ \\
$27.7 \mu \mathrm{m}$ \\
broad
\end{tabular} & $\begin{array}{c}+ \\
\& \\
3 \text { in no. in } \\
\text { each zone }\end{array}$ & $\begin{array}{c}\text { Elongated } \\
\text { to barrel }\end{array}$ & $\begin{array}{c}11.1-16.6 \\
\mu \mathrm{m} \text { long } \\
\& 16.6- \\
33.3 \mu \mathrm{m} \\
\text { broad }\end{array}$ & \begin{tabular}{|c|} 
Elongated, \\
quadrangular, \\
to pentagonal
\end{tabular} & $\begin{array}{c}11.1-27.7 \\
\mu \mathrm{m} \text { long \& } \\
11.1-16.6 \\
\mu \mathrm{m} \text { broad }\end{array}$ & $\begin{array}{c}66.6-88.8 \\
\mu \mathrm{m} \text { long \& } \\
155.4-177.6 \\
\mu \mathrm{m} \text { broad }\end{array}$ & - & - \\
\hline 5. & $\begin{array}{c}\text { P. greggii } \\
\text { Engelmann } \\
(3 \text { needles per } \\
\text { fascicle })\end{array}$ & $\begin{array}{l}\text { Thick } \\
\text { walled }\end{array}$ & $\begin{array}{c}\text { Oval, } \\
\text { isodiametric, } \\
\text { elongated to } \\
\text { rectangular }\end{array}$ & $\mid \begin{array}{c}5.55-11.1 \mu \mathrm{m} \\
\text { long \& } 5.55- \\
11.1 \mu \mathrm{m} \\
\text { broad }\end{array}$ & \begin{tabular}{|c|} 
Oval, \\
elongated to \\
quadrangular \\
to pentagonal
\end{tabular} & $\begin{array}{c}5.55-11.1 \\
\mu \mathrm{m} \text { long } \& \\
5.55-11.1 \\
\mu \mathrm{m} \text { broad }\end{array}$ & $\begin{array}{c}\text { Oval } \\
\text { elongated } \\
\text { quadrangular, } \\
\text { penta to } \\
\text { octagonal }\end{array}$ & \begin{tabular}{|c|}
$11.1-$ \\
$27.7 \mu \mathrm{m}$ \\
long \& \\
$11.1-$ \\
$33.3 \mu \mathrm{m}$ \\
broad
\end{tabular} & $\begin{array}{c}+ \\
\& \\
2 \text { in no. in } \\
\text { each zone }\end{array}$ & $\begin{array}{c}\text { Elongated } \\
\text { to barrel }\end{array}$ & $\begin{array}{c}11.1-16.6 \\
\mu \mathrm{m} \text { long } \\
\& 16.6- \\
27.7 \mu \mathrm{m} \\
\text { broad }\end{array}$ & \begin{tabular}{|c|} 
Elongated, \\
quadrangular, \\
to pentagonal
\end{tabular} & $\begin{array}{c}11.1-27.7 \\
\mu \mathrm{m} \text { long \& } \\
11.1-16.6 \\
\mu \mathrm{m} \text { broad }\end{array}$ & 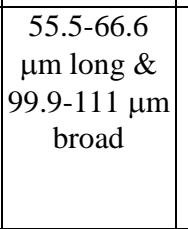 & - & - \\
\hline
\end{tabular}

ENDO. = ENDODERMIS; PERI. = PERICYCLE; R.D. = RESIN DUCT; V.B. = VASCULAR BUNDLE; N.D. = NOT DISTINCT; L.D. = LESS DISTINCT; += PRESENT; 
Plate.1 Pinus canariensis fig.(1) Habit,fig.(2) a twig with spur shoot, fig.(3) T.S. of basal region of dwarf shoot X120. fig.(4) T.S. of middle region of dwarf shoot X120. fig(5)T.S. of upper region of dwarf shoot X120, P. densiflora fig.(6) Habit, fig.(7) a twig with spur shoot, fig.(8) T.S. of basal region of dwarf shoot X120. fig.(9) T.S. of middle region of dwarf shoot X120. fig(10)T.S. of upper region of dwarf shoot X120, P. echinata fig.(11) Habit,fig.(12) a twig with spur shoot, fig.(13) T.S. of basal region of dwarf shoot X120. fig.(14) T.S. of middle region of dwarf shoot X120. fig(15)T.S. of upper region of dwarf shoot X120.
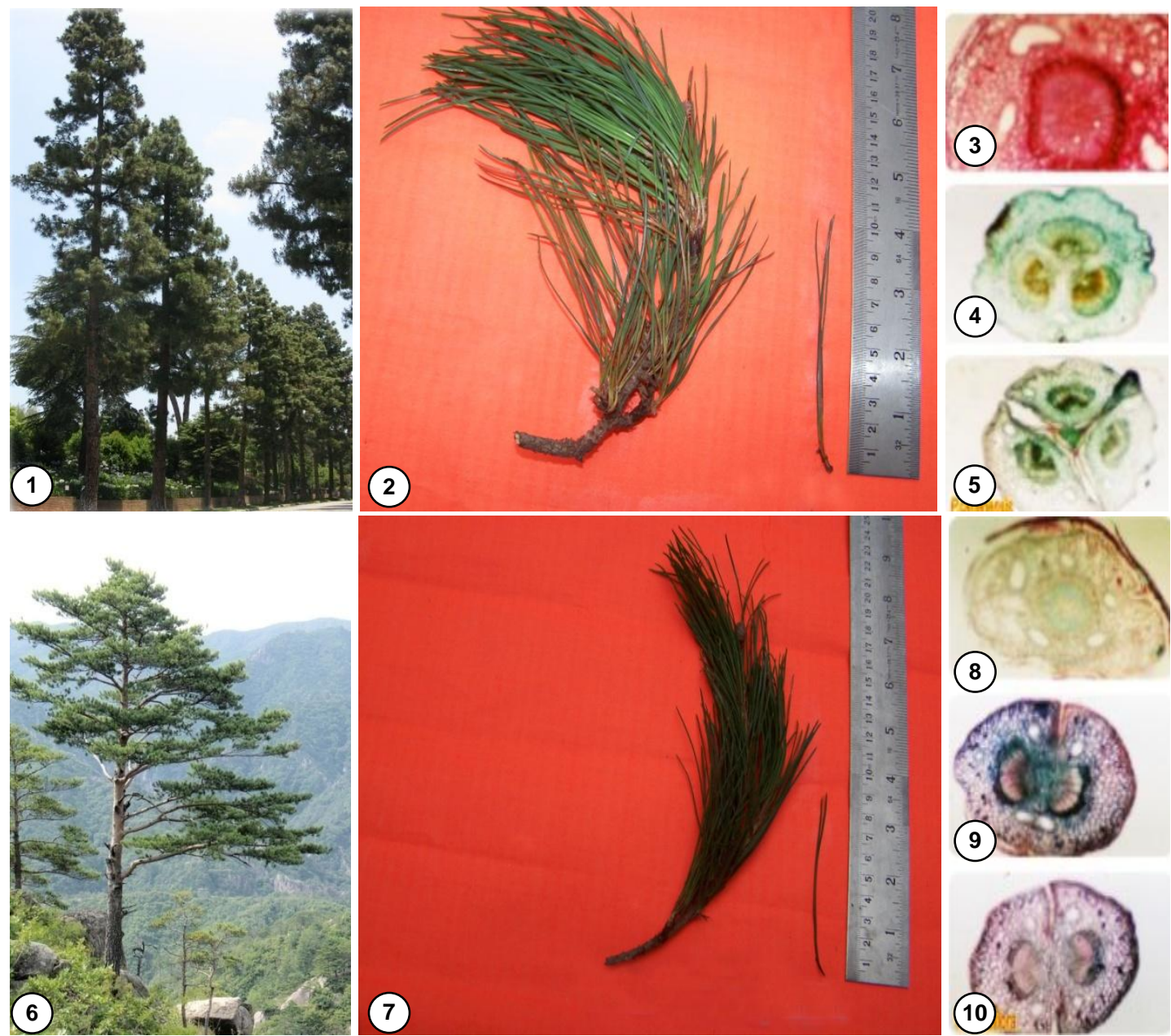

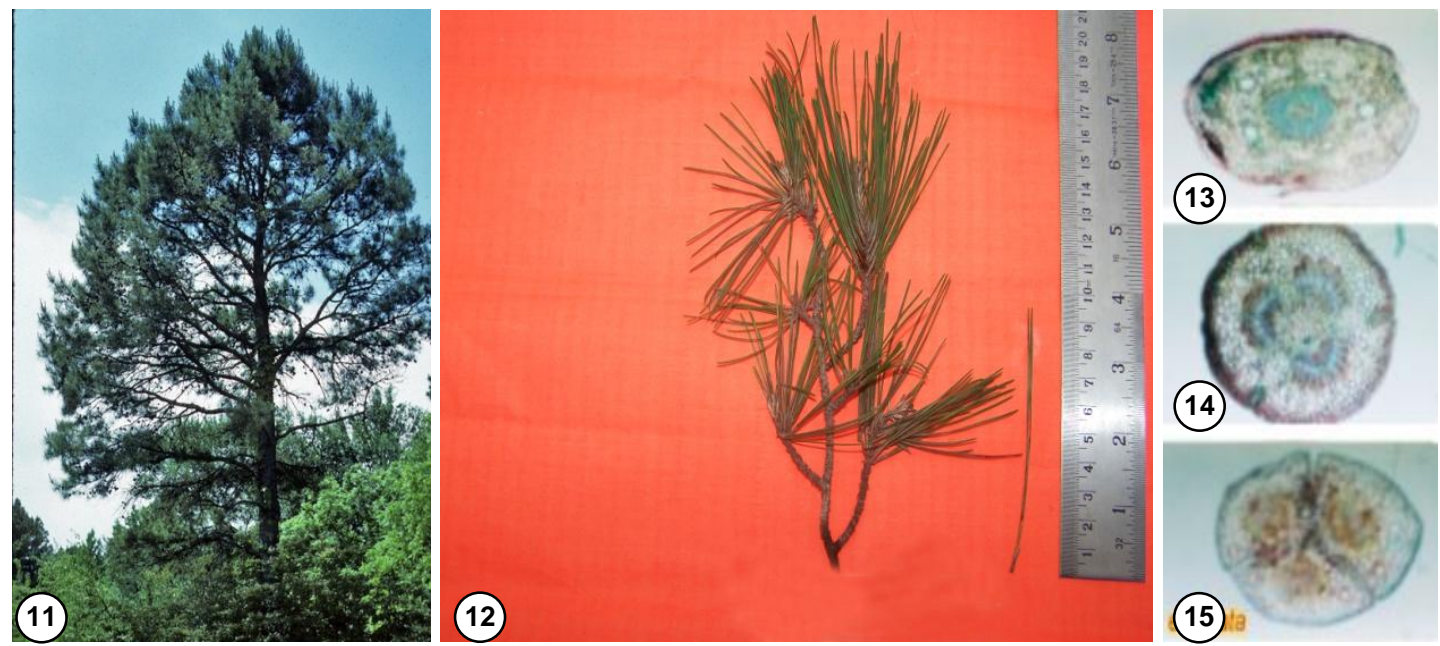

Plate 2 P.engelmanii fig.(1) Habit, fig.(2) a twig with spur shoot, fig.(3) T.S. of basal region of dwarf shoot X120. fig.(4) T.S. of middle region of dwarf shoot X120. fig(5)T.S. of upper region of dwarf shoot X120, P. greggii fig.(6) Habit,fig.(7) a twig with spur shoot, fig.(8) T.S. of basal region of dwarf shoot X120. fig.(9) T.S. of middle region of dwarf shoot X120. fig(10)T.S. of upper region of dwarf shoot X120.
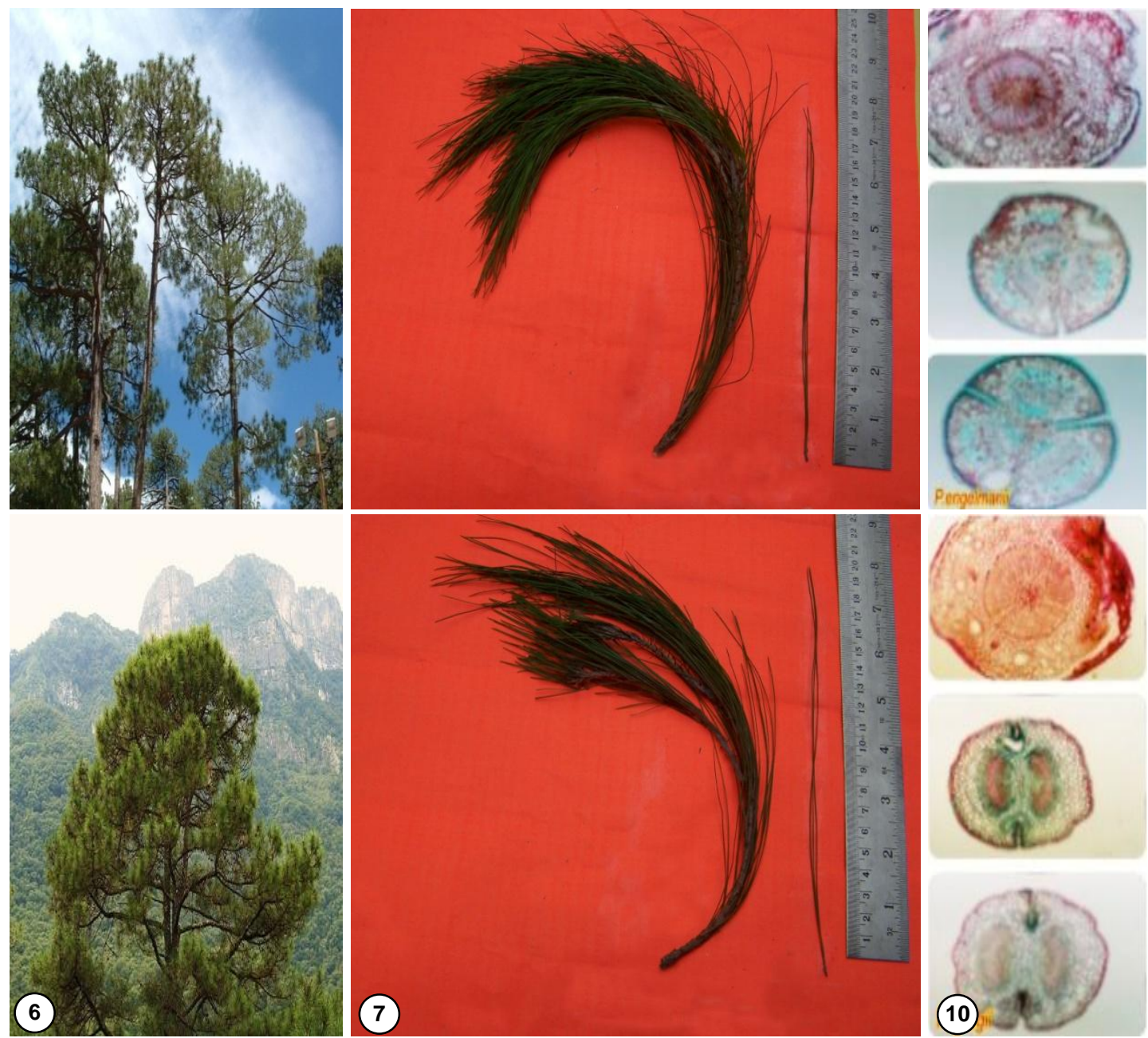
The size of inner cortical cells may also vary among the species. 11.1-16.6 $\mu \mathrm{m}$ in long and 11.1-22.2 $\mu \mathrm{m}$ in broad in P. densiflora (Plate 1, Fig. 8,Table 1) and $P$. greggii (Plate 2, Fig. 8,Table 1). 11.1-22.2 $\mu \mathrm{m}$ in long and 11.1-16.6 $\mu \mathrm{m}$ in broad in $P$. canariensis (Plate 1, Fig. 3, Table 1). P. echinata (Plate 1, Fig. 13, Table 1) has 5.55$16.6 \mu \mathrm{m}$ in long and 5.55-16.6 $\mu \mathrm{m}$ in broad while P. engelmanii (Plate 2, Fig. 3, Table 1) shows 11. 1-16.6 $\mu \mathrm{m}$ in long and 11.1-27.7 $\mu \mathrm{m}$ in broad. Resin ducts are absent in this zone in case of $P$. echinata (Plate 1, Fig. 13) and $P$. engelmanii (Plate 2, Fig. 3). In $P$. canariensis (Plate 1, Fig. 3), these are numerous in number, large, distributed here and there, lined by thin walled, single layered of epithelial cells, placed intermarginally while these are few in number, small, two layered of epithelial cells, placed inter marginally in case of $P$. densiflora (Plate 1, Fig. 8) and P. greggii (Plate 2, Fig. 8).

Barbaros Yaman (2007) explained that the anatomical properties of the wood of $P$. sylvestris var. compacta and compares them with typical $P$. sylvestris. The woods of these taxa have the same qualitative anatomical features but most of the quantitative anatomical characteristics show significant differences.

The vascular cylinders characterized by having well marked secondary growth, due to which large amount of secondary wood is formed. Amount of secondary wood is less than the amount of cortex observed in $P$. canariensis (Plate 1, Fig. 3) which shows affinity with $P$. densiflora (Plate 1, Fig. 8), P. echinata (Plate 1, Fig. 13), P. engelmanii (Plate 2, Fig. 3) and P. greggii (Plate 2, Fig. 8). The size of vascular cylinders may also vary among the species.

Maximum size of vascular cylinders i.e. 321.9-333 $\mu \mathrm{m}$ in long and 277.5-288.6 $\mu \mathrm{m}$ in broad observed in P. canariensis (Plate 1, Fig. 3,Table 1) which shows close affinity with $P$. engelmanii (Plate 2, Fig. 3,Table 1) i.e. $321.9-333 \mu \mathrm{m}$ in long and 210.9-222 $\mu \mathrm{m}$ in broad. Lowest size of vascular cylinders i.e. $155.4-166.5 \mu \mathrm{m}$ in long and 166.5 to $177.6 \mu \mathrm{m}$ in broad observed in $P$. greggii (Plate 2, Fig. 8,Table 1) which shows close affinity with $P$. echinata (Plate 1, Fig. 13 ,Table 1) i.e. $222-233 \mu \mathrm{m}$ in long and $177-188 \mu \mathrm{m}$ in broad. 266.4-277.5 $\mu \mathrm{m}$ in long and 244.2-255.3 $\mu \mathrm{m}$ in broad observed in P. densiflora (Plate 1, Fig. 8,Table 1). Resin ducts are absent in wood region observed in P. densiflora (Plate 1, Fig. 8), P. engelmanii (Plate 2, Fig. 3) and $P$. greggii (Plate 2, Fig. 8) while these are present in $P$. canariensis (Plate 1, Fig. 3) and P. echinata (Plate 1, Fig. 13). Amount of pith is centrally located but less in amount observed in P. canariensis (Plate 1, Fig. 3), P. densiflora (Plate 1, Fig. 8), P. echinata (Plate 1, Fig. 13) and P. engelmanii (Plate 2, Fig. 3) while these are centrally located but least in amount observed in $P$. greggii (Plate 2, Fig. 8).

The middle region of dwarf shoot shows differentiation in structure. The whole structure may be circular or oval in outline but differentiation may occur among the species. The middle region of the dwarf shoots covered with thick walled cuticle observed in P. canariensis (Plate 1, Fig. 4), $P$. echinata (Plate 1, Fig. 14) whereas these are thin walled observed in $P$. densiflora (Plate 1, Fig. 9) and P. greggii (Plate 2, Fig. 9). P. engelmanii (Plate 2, Fig. 4) has cuticle in the form of scale leaves. The shape and size of epidermal cells may also vary in middle region of the dwarf shoots. The cells of epidermis are isodiametric, elongated to rectangular in $P$. canariensis (Plate 1, Fig. 4) whereas in P. densiflora (Plate 1, Fig. 9), these are oval, elongated and quadrangular. Oval, isodiametric, quadrangular to 
elongated in P. echinata (Plate 1, Fig. 14). In $P$. engelmanii (Plate 2, Fig. 4), the epidermis is not distinct. In $P$. greggii (Plate 2, Fig. 9), these are oval isodiameteric, elongated to rectangular in shape. The cells of epidermis are 5.55-11.1 $\mu \mathrm{m}$ in long and 5.55-16.6 $\mu \mathrm{m}$ in broad observed in $P$. densiflora (Plate 1, Fig. 9, Table 2). 11.1$16.6 \mu \mathrm{m}$ in long and 11.1-16.6 $\mu \mathrm{m}$ in broad in $P$. canariensis (Plate 1, Fig. 4,Table 2) and $P$. greggii (Plate 2, Fig. 9, Table 2). 5.55-11.1 $\mu \mathrm{m}$ in long and 5.55-11.1 $\mu \mathrm{m}$ in broad observed in $P$. echinata (Plate 1, Fig. 14, Table 2).

The very important significant feature of middle region of the dwarf shoots is the distinction of cortex as well as vascular region into two, three, four, five, etc. regions depending upon their needles per fascicle, is already begins by the broadening of medullary rays from pith to cortex and resin ducts are few in number. The wood region is present in the form of vascular cylinders. $P$. canariensis (Plate 1, Fig. 4, Table 2) has 111-122.1 $\mu \mathrm{m}$ in long and 177.6-199.8 $\mu \mathrm{m}$ in broad vascular cylinders. $99.9-111 \mu \mathrm{m}$ in long and 177.6-199.8 $\mu \mathrm{m}$ in broad in $P$. densiflora (Plate 1, Fig. 9, Table 2), 88.8$99.9 \mu \mathrm{m}$ in long and 188.7-199.8 $\mu \mathrm{m}$ in broad in P. echinata (Plate 1, Fig. 14,Table 2), 111-133.2 $\mu \mathrm{m}$ in long and 188.7-222 $\mu \mathrm{m}$ in broad in $P$. engelmanii (Plate 2, Fig. 4,Table 2) and 66.6-77.7 $\mu \mathrm{m}$ in long and $111-133.2 \mu \mathrm{m}$ in broad vascular cylinders observed in $P$. greggii (Plate 2, Fig. 9, Table 2).

The feature of secondary wood appears to be an important feature and can be used as a character for identification of species. Another significant feature of the dwarf shoot in the middle region in which fused resin ducts may also present (Plate 1, Fig. 9, Plate 2, Fig. 4).
The cross section of the dwarf shoot at upper region is almost circular in outline with clearly distinct two independent semicircular in P. densiflora (Plate 1, Fig. 10) and $P$. greggi (Plate 2, Fig. 10) whereas three independent triangular zones observed in $P$. canariensis (Plate 1, Fig. 5), P. echinata (Plate 1, Fig. 15) and P. engelmanii (Plate 2, Fig. 5). Each zone is remain united at inner face while free at the outerface. Each zone is rather independent except centrally at inner face and bounded by single layer epidermis.

Annual shoot elongation decreased with increasing tree size. This is described by Moorby and Wareing (1963) for trees of different ages and sizes.

Ewers and Schmid (1981) demonstrated that the variability in leaf longevity in several pine species may involve responses to environmental conditions as well as variability within a genotype. Weideman (1939) provided data for ponderosa pine to support the same conclusion.

The very important significant feature of the dwarf shoot at upper region is the distinction of needles into two, three, four, five, etc. zones. Each zone is remains united at innerface while free at the outerface. Each zone has an epidermis, hypodermis, cortex is present in the form of mesophylls, endodermis, pericycle and centrally located vascular cylinders. Here pith is altogether absent (Plate 1, Figs.5, 10, 15, Plate 2, Figs. 5, 10, Table 3). Moreover, shape and size of epidermal cells, amount of cortex, number and position of resin ducts, amount of secondary wood, etc. may vary from species to species of Pinus. These important feature can use as a character for identification of species. The study revealed that anatomical variations in different regions of dwarf shoots can be taxonomically very helpful in identification of various species of Pinus. 


\section{References}

Antony, F., Schimleck, L.R. and Daniels, R.F. 2012. A comparison of early wood-latewood demarcation methods - A case study in loblolly Pine. IAWA J., 33: 187-195.

Boratynska, K. and Boratynski, A. 2003. Anomalous needle numbers on dwarf shoots of Pinus mugo and Pinus uncinata (Pinaceae). Acta Soc. Bot. Pol., 72: 139-148.

Boratynski, A., Dubicki, A., Iszkulo, G., Jasinska, A.K., Boratynska, K., Piorkowska, M. and Marcysiak, K. 2011. How environmental signals affect frequency of three-needle dwarf shoots on Pinus mugo? Polish J. Environ. Stud., 20: 45-52.

Borthwick, A.W. 1899a. On the development of quadrifoliar spurs in Pinus laricio. Poir. Trans. Proc. Bot. Soc. Edinb., 21: 150-153.

Buchholz, J.T. and Gray, N.E. 1948. A taxonomic revision of Podocarpus-I. The section of the genus and their sub divisions with special reference to leaf anatomy. J. Arnold Arboretum, 29: 4963.

Chaturvedi, S. 1993. Anatomy of needles of two species of Pinus L. Proc. Nat. Acad. Sci. India, 63(II)IV: 401-4.

Dallimore, W. and Jackson, A.B. 1966. A handbook of coniferae including Ginkgoaceae, London.

Debazac, E.F. 1962. Note sur le bourgeon interfasiculaire et les aiguilles surnumeraires chez les. Pins. Bull. Soc. Bot. Fr., 109: 1-5.

Doak, C.C. 1935. Evolution of foliar types, dwarf shoots and cone scales in Pinus. Illinois Biol. Monogr., 13: 1-106.

Donaldson, L.A., Grace, J. and Downes, G.M. 2004. Within-tree variation in anatomical properties of compression wood in Radiata Pine. IAWA J., 25:
253-271.

Durell, L.W. 1916. Notes on some North American conifers based on leaf characters. Iowa Acad. Sci. Proc., 23: 519-582.

Eom, Y.G. and Chung, Y.J. 1994. Tumor wood anatomy in Korean red pine (Pinus densiflora) IAWA J., 15: 149155.

Esteban, L.G., Gasson, P., Climent, J.M., Palacios, P. de and Guindeo, A. 2005. The wood of Pinus canariensis and its resinous heartwood. IAWA J., 26: 6977.

Ewers, F.W. 1982. Developmental and cytological evidence for mode of origin of secondary phloem in needle leaves of Pinus longaeva (Bristlecone pine) and Pinus flexilis. Bot. Jahrb. Syst., 103: 59-88.

Ewers, F.W. 1983. The determinate and indeterminate dwarf shoots of Pinus longaeva (bristlecone pine). Can. J. Bot., 61: 2280-2290.

Ewers, F.W. and Aloni, R. 1987. Seasonal secondary growth in needle leaves of Pinus strobus and Pinus brutia. Amer. J. Bot., 74: 980-987.

Ewers, F.W. and Schmid, R. 1981. Longevity of needle fascicles of Pinus longaeva (bristlecone pine) and other Northern American pines. Oecologia, 51: 107-115.

Ewers, F.W. and Schmid, R. 1985. The fate of the dwarf shoot apex in bristlecone pine (Pinus longaeva). Amer. J. Bot., 72: 509-513.

Florin, R. 1940. The tertiary fossil conifers of South Chile and their phytogeographical significance, with a review of the fossil conifers of southern land, Kgl. Svenka Ventensk Acad. Handl. III 10: 1-588, Stockholm.

Florin, R. 1951. Evolution of Cordaites and Conifers. Acta Horti Bergiani, 15: 
285-388.

Florin, R. 1958. Notes on the systematic of the Podocarpaceae. Acta Horti Bergiani, 17: 403-411.

Florin, R. 1963. The distribution of conifer and Taxad genera in time and space. Acta Horti Bergiani, 20: 121-312.

Foster, A.S. 1938. Structure and growth of the shoot apex in Ginkgo biloba. Bull. Torrey Bot. Club., 65: 531-536.

Fulling, E.H. 1934. Identification by leaf structure of the species of Abies cultivated in the United States, Torrey Bot. Club. Bul., 61: 497-524.

Gabilo, E.M. and Mogensen, H.L. 1973. Foliar initiation and the fate of the dwarf shoot apex in Pinus monophylla. Amer. J. Bot., 60: 671677.

Geylor, H.T. 1867. Uber den gefassbiindelverlauf in den laubblattrengionen der coniferen. Jahrb. Wiss. Bot., 6: 55-208.

Ghouse, A.K.M. and Yunus, M. 1972. Leaf anatomy in the classification of Indian conifers. Research trends in plant anatomy (K.A. Chowdhery commemoration volume), Tata $\mathrm{Mc}$ Graw Hill Pub. Company Ltd., Bombay, New Delhi.

Hafren, J., Daniel, G. and Westermark, U. 2000. The distribution of acidic and esterified pectin in cambium, developing xylem and mature xylem of Pinus sylvestris. IAWA J., 21: 157168.

Hanawa, J. 1967. Growth and development in the shoot apex of Pinus densiflora II. Ontogeny of the dwarf shoot and the lateral branch. Bot. Mag., (Tokyo) 80: 248-256.

Harlow, W.M. 1931. The identification of the pines of United States native and introduced by needle structure. N.Y. State Col. Forestry, Syracuse Univ. Tech. Pub. 32.
Henry, A. 1837. Betrag zur Kenntniss der Laublenospen. Nova Acta Leop.-Carol. Akad. Naturf. Verh., 19: 87-114.

Heuret, P., Meredieu, C., Coudurier, T., Courdier, F. and Barthelemy, D. 2006. Ontogenetic trends in the morphological features of main stem annual shoots of Pinus pinaster (Pinaceae). Amer. J. Bot., 93: 1577-1587.

Johansen, D.A. 1940. Plant Microtechnique, Mc Graw Hill Book Company.

Konar, R.N. 1963. Anatomical studies on Indian pines with special reference to Pinus roxburghii Sarg. Phytomorphol., 13: 388-402.

Lee, P.W. and Eom, Y.G. 1988. Anatomical comparison between compression wood and opposite wood in a branch of Korean Pine (Pinus koraiensis). IAWA Bull., n.s. 9: 275-284.

Lim, D.O. and Soh, W.Y. 1997. Cambial development and tracheid length of dwarf pines (Pinus densiflora and Pinus thunberghii). IAWA J., 18: 301-310.

Marco, H.F. 1939. The anatomy of spruce needles. J. Agr. Res., 58: 357-368.

Mehra, P.N. 1988. Indian Conifers, Gnetophytes Phylogeny of Gymnosperms, pp. 121-143.

Moorby, J. and Wareing, P.F. 1963. Ageing in woody plants. Ann. Bot., 27: 291-308.

Nair, H., Butterfield, B. and Jackson, S. 2009. Are rays and resin canals causal sites for intra-ring checking in the wood of Pinus radiata? IAWA J., 30: 189-198.

Orr, M.M. 1944. The leaf anatomy of Podocarpus. Trans. Proc. Bot. Soc. Edinburg, 34: 1-54. 
Owens, J.N. and Molder. 1977. Development of long-shoot terminal buds of Western white pine (Pinus monticola). Can. J. Bot., 55: 1308-1381.

Prat, H. 1930. Note sur les pousses courtes du pin maritime. Bull. Sta. Biol. D'Archachon, 27: 1-20.

Reich, P.B., Oleksyn, J., Modrzynski, J. and Tjoelker, M.G. 1996. Evidence that longer needle retention of spruce and pine populations at high elevations and high latitudes is largely a phenotypic response. Tree Physiol., 16: 643-647.

Sache, J.A. 1955. Dwarf shoot ontogeny in Pinus lambertiana. Amer. J. Bot., 42: 784-792.

Sache, J.A. 1954. Structure and seasonal activity of the shoot apices of Pinus lambertiana and Pinus ponderosa. Amer. J. Bot., 41: 749759.

Schneider, W. 1913. Vergleichend morphologische unter suchungen uber die Kurztriebe einige Arten Von Pinus. Flora, 105: 385-446.

Thomson, R.B. 1914. The spur shoot of the pines. Bot. Gaz., 57: 362-385.

Weidman, R.H. 1939. Evidence of racial influence in a 25-year test for ponderosa pine. J. Agric. Res., 59: 855-887.

Yadun, S.L. 1998. The relationship between growth-ring width and ray density and ray height in cell number in the early wood of Pinus halepensis and Pinus pinea. IAWA J., 19: 131-139.

Yaman, B. 2007. Comparative wood anatomy of Pinus sylvestris and its var. compacta in the West Black Sea region of Turkey. IAWA J., 28: 75-81.

Zhang, C., Fujii, T., Abe, H., Fujiwara, T., Fujita, M. and takabe, K. 2008. Anatomical features of radial resin canals in Pinus densiflora. IAWA J., 29: 179-187.

\section{How to cite this article:}

Dildar Husain. 2016. Morphology and Anatomy of Dwarf Shoots of Some Exotic Species of Pinus Linn. in Kumaon Hills, Western Himalayas. Int.J.Curr.Microbiol.App.Sci. 5(9): 219-233. doi: http://dx.doi.org/10.20546/ijcmas.2016.509.025 